\title{
Aplicações educacionais com óculos de realidade virtual: um Mapeamento Sistemático
}

\author{
Layon Martins Fonseca, Joice Lee Otsuka
}

${ }^{1}$ Departamento de Computação - Universidade Federal de São Carlos (UFSCAR)

Caixa Postal 676 1365-905 - São Carlos - SP- Brasil

layonmartins03@gmail.com, joicedufscar.br

\begin{abstract}
This work presents a systematic mapping, aiming to identity the state of the art in the Virtual Reality applied to Education, focusing on Virtual Reality glasses. This study aims to identify which glasses, interaction techniques and devices, application areas, softwares and evaluation methods were most used in the analysed works.
\end{abstract}

Resumo. Este trabalho apresenta um mapeamento sistemático com objetivo de identificar o estado da arte na área de Realidade Virtual aplicada à Educação com foco em solução de baixo custo que utilizam óculos de Realidade Virtual. Este estudo busca identificar quais óculos, técnicas e dispositivos de interação, áreas de aplicação, softwares e formas de avaliação foram mais utilizados nos trabalhos analisados.

\section{Introdução}

A Realidade Virtual - RV é uma tecnologia na computação que fornece ao usuário a possibilidade de "estar" em outros lugares sem precisar sair do seu local atual por meio de uma visualização tridimensional. Basicamente ela "engana" os sentidos humanos, principalmente a visão, dando um nível de imersão e interação que tornam possível esta experiência.

O foco deste trabalho são os óculos de RV, também conhecidos como "Head Mounted-Display- HMD”. São dispositivos que projetam a imagem frente aos olhos do usuário. Nos últimos anos, os óculos de RV tiveram uma boa repercussão com lançamentos de modelos de baixo custo, como "Oculus Rift", "Google Cardboard" etc.

Este cenário abre uma gama de possibilidades de estudos sobre as propriedades dos óculos de RV, principalmente referentes à imersão e à interação, que podem ser exploradas no desenvolvimento de novas aplicações educacionais.

De acordo com [Pantelidis 2009], a RV tem o potencial de fazer a diferença em todos os níveis de educação, motivando, encorajando e estimulando os processos de ensinoaprendizagem. Os alunos podem interagir com o ambiente de aprendizagem com sentimento de fazer parte, de estar dentro.

Dessa forma, este artigo tem como objetivo apresentar e discutir os principais resultados obtidos em um mapeamento sistemático identificando o estado da arte na área de RV aplicada à Educação, com foco em solução de baixo custo que utilizam óculos de RV. O restante do artigo está organizado da seguinte forma: a seção 2 apresenta trabalhos relacionados. Na seção 3 é apresentado o planejamento do mapeamento sistemático e na 
VI Congresso Brasileiro de Informática na Educação (CBIE 2017)

Anais do XXVIII Simpósio Brasileiro de Informática na Educação (SBIE 2017)

seção 4 são apresentadas considerações sobre a condução do mapeamento. Na seção 5 são apresentados os principais resultados obtidos. Por fim, na seção 6, é apresentada uma discussão e as considerações finais.

\section{Trabalhos Relacionados}

Kawakami et al. (2015) apresentam uma revisão sistemática que procura identificar aplicações de "Ambientes Virtuais Tridimensionais - AV 3D" para apoiar o processo de aprendizagem de crianças hospitalizadas. Eles propõem duas questões de pesquisa: (1) "Quais são as estratégias de interação adotadas nos AV 3D para estimular o aprendizado de crianças hospitalizadas ou que apenas estejam afastadas do ambiente escolar por motivo de doença?" e (2) "Quais fatores humanos são considerados na concepção e aplicação dos AV 3D voltados para assistência às crianças afastadas do ambiente escolar por motivo de doença (hospitalizadas ou não)?”. Os autores concluem que há um notável interesse de pesquisadores em conceber AV 3D para crianças hospitalizadas e que vários trabalhos adotam estratégias de interação que empregam técnicas de Realidade Virtual e Aumentada com diferentes níveis de imersão. Também os aspectos sociais e emocionais são usados como fatores humanos para estes tipos concepção de AV 3D.

Davis et al. (2014) apresentam uma revisão sistemática na área de cybersickness (tontura, mal-estar etc.), com objetivo de medir diversos sintomas apresentados ao utilizar óculos de RV. Os autores propõem as questões de pesquisa: (1) "Existe uma medida fisiológica para quantificar a sensibilidade de um indivíduo ao cybersickness?", (2) "Como a cybersickness atualmente é detectada e medida na RV?" e (3) "Existe um método econômico de detectar cybersickness na RV?". Eles concluem que a maioria das medidas existentes depende de autorrelatos ou sistemas de medição objetiva mais caros e complexos. O desenvolvimento de medidas objetivas para o cybersickness é um passo importante na compreensão das causas e efeitos que podem ter sobre os participantes, além de auxiliar nas tentativas de melhorar o design das tecnologias envolvidas e dos ambientes em desenvolvimento.

Proenca et al. (2017) realizaram um estudo baseado em uma revisão sistemática com o objetivo de avaliar a usabilidade de AV 3D educativos ou de treinamento (aplicados em RV) e propor um quadro com diretrizes aplicáveis a estes ambientes. Eles concluem que a RV vem sendo amplamente utilizada como ferramenta de auxílio pedagógico, e apresentam-se como alternativas mais lúdicas, colaborativas, com uma linguagem mais próxima do aluno, promovendo maior envolvimento emocional e motivação. Já para treinamento, são muito mais realistas, com movimentos precisos para refletir a usabilidade de um ambiente real. Apresentam também dois quadros com dados quantitativos de 10 diretrizes (cada um) para AV 3D de educação e treinamento.

Os trabalhos relacionados apresentam revisões sistemáticas relevante à $\mathrm{RV}$, um voltado para aprendizagem de crianças hospitalizadas, outro sobre cybersickness causadas ao utilizar óculos de RV e o último uma avaliação de usabilidades em AV 3D para propor diretrizes aplicáveis ao desenvolvimento de aplicações de educação e treinamento em RV. Dessa forma, o presente trabalho traz uma nova contribuição para área, apresentando um mapeamento sistemático das pesquisas que vêm sendo realizadas (2013-2017) na área de RV aplicada à Educação, com foco em soluções baseadas em óculos de RV. 
VI Congresso Brasileiro de Informática na Educação (CBIE 2017)

Anais do XXVIII Simpósio Brasileiro de Informática na Educação (SBIE 2017)

\section{Planejamento do mapeamento}

Este mapeamento seguiu a método proposto por [Kitchenham and Charters 2007] dividindo-se em três etapas principais: Planejamento, Condução e Extração dos Resultados. Todo o planejamento foi documentado no protocolo de definições das questões norteadoras da pesquisa: objetivo, questões de pesquisa, estratégia de busca, seleção dos trabalhos (critérios de inclusão e exclusão).

O objetivo da pesquisa foi identificar o estado da arte na área de RV aplicada à Educação, tendo como foco soluções de baixo custo (óculos de RV), resultando em uma visão geral dos tipos de aplicações educacionais que vêm sendo propostas; as soluções adotadas; as técnicas de interação utilizadas para movimentação e manipulação dentro do AV 3D; formas de avaliação dos trabalhos (analíticas, empíricas) e áreas de aplicação.

Baseado nos objetivos expostos, foram propostas seis questões de pesquisa. As questões e respectivas motivações são apresentadas na Tabela 1.

Tabela 1. Questões de pesquisa

\begin{tabular}{|l|l|}
\hline Questões de pesquisa & Motivação principal \\
\hline QP1. Qual óculos de RV foi utilizado? & $\begin{array}{l}\text { Identificar qual modelo de óculos de RV foi utilizado como, Google Cardboard, } \\
\text { Oculus Rift etc. }\end{array}$ \\
\hline $\begin{array}{l}\text { QP2. Qual software foi utilizado para desen- } \\
\text { volver a aplicação de RV? }\end{array}$ & $\begin{array}{l}\text { Identificar os softwares utilizados, na modelagem, desenvolvimento, } \\
\text { programação etc. }\end{array}$ \\
\hline $\begin{array}{l}\text { QP3. Foram utilizados dispositivos além dos } \\
\text { óculos de RV para melhorar a interação? }\end{array}$ & $\begin{array}{l}\text { Identificar os dispositivos físicos para entrada de informação utilizados junto com } \\
\text { óculos de RV como, luva de dados, joystick, teclado e mouse etc. }\end{array}$ \\
\hline $\begin{array}{l}\text { QP4. Quais técnicas de interação foram uti- } \\
\text { lizadas? }\end{array}$ & $\begin{array}{l}\text { Determinar se foi utilizada alguma técnica específica para interagir com a } \\
\text { aplicação, como se locomover ou selecionar e manipular objetos dentro do MV. }\end{array}$ \\
\hline QP5. Qual área de aplicação? & $\begin{array}{l}\text { Identificar quais áreas do conhecimento têm investido no desenvolvimento de sis- } \\
\text { temas com base em óculos de RV (exatas, humanas, saúde etc). }\end{array}$ \\
\hline $\begin{array}{l}\text { QP6. Foram utilizados métodos de } \\
\text { avaliação? }\end{array}$ & $\begin{array}{l}\text { Identificar se os trabalhos passaram por algum tipo de avaliação analítica ou } \\
\text { empírica. }\end{array}$ \\
\hline
\end{tabular}

Após definir as questões de pesquisa, foi construída uma string de busca genérica baseada em [Brereton et al. 2007]. Primeiramente, definiu-se termos principais relacionados com as questões de pesquisa. Em segundo momento, identificou-se os termos relacionados ou sinônimos (derivados) destes termos principais conectando-os pelo operador OR e, por fim, conectando estes termos com o operador AND.

Inicialmente foi realizado um teste piloto aplicando os termos principais "virtual reality" AND "head-mounted display" AND "education" e analisando os trabalhos retornados, resumos, palavras-chave para definir os termos derivados conforme mostra a Tabela 2.

Tabela 2. Termos utilizados na string de busca

\begin{tabular}{|l|l|}
\hline Termo principal & Termos derivados \\
\hline Realidade Virtual & ("virtual reality") \\
\hline Óculos de RV & $\begin{array}{l}\text { ("virtual reality glasses" OR "stereotypic glasses" OR "head-mounted displays" OR HMD OR "virtual rea- } \\
\text { lity headset" OR "rift" OR "samsung gear" OR "htc vive" OR “cardboard" OR "daydream") }\end{array}$ \\
\hline Educação & ("educat" OR "learning" OR “teaching" OR "training") \\
\hline
\end{tabular}

Para a seleção dos trabalhos, foram definidos critérios de inclusão e exclusão para que fosse possível filtrar os que iriam responder às questões de pesquisa (Tabela 3).

$\mathrm{Na}$ estratégia de busca foram selecionadas fontes e revistas consideradas relevantes para área, além da definição dos filtros, linguagens e período de publicação, apresentados na Tabela 4. 
VI Congresso Brasileiro de Informática na Educação (CBIE 2017)

Anais do XXVIII Simpósio Brasileiro de Informática na Educação (SBIE 2017)

Tabela 3. Critérios de Inclusão e Exclusão

\begin{tabular}{|l|l|}
\hline $\begin{array}{l}\text { Critérios } \\
\text { de In- } \\
\text { clusão }\end{array}$ & $\begin{array}{l}\text { CI1. Serão incluídos trabalhos que apresentam aplicações educacionais ou de treinamento que utilizam óculos de RV. } \\
\text { CI2. Serão incluídos trabalhos que fazem análises ou comparações de interação ou imersão com RV que estejam } \\
\text { relacionados com a educação. } \\
\text { CI3. Serão incluídos trabalhos que fazem estudos sobre aplicações RV com a educação. }\end{array}$ \\
\hline $\begin{array}{l}\text { CE1. Serão excluídos trabalhos que apenas comentem sobre a utilização de óculos de RV e não apresentem alguma } \\
\text { característica. }\end{array}$ \\
$\begin{array}{l}\text { CE2. Serão excluídos trabalhos que não são de ambientes tridimensionais. } \\
\text { de Ex- } \\
\text { clusão }\end{array}$ & $\begin{array}{l}\text { CE3. Serão excluídos trabalhos com foco em tratamento de doença, reabilitação, terapia, na área de saúde em geral } \\
\text { sem o contexto educacional. } \\
\text { CE4. Serão excluídos trabalhos que apresentam a mesma aplicação de outro artigo. }\end{array}$ \\
\hline
\end{tabular}

Tabela 4. Estratégia de busca

\begin{tabular}{|l|l|}
\hline Fontes & $\begin{array}{l}\text { Biblioteca Digital da ACM, Biblioteca Digital do IEEE, Scopus, Springer, Elsiever, SBGames, IHC, SVR, SBIE, } \\
\text { RBIE, Computer and Education. }\end{array}$ \\
\hline Filtros & Título, Resumo, Palavras-chave. \\
\hline Linguagem & Português e Inglês. \\
\hline Período & A partir de 2013 (inclusive). \\
\hline
\end{tabular}

\section{Condução do mapeamento}

$\mathrm{Na}$ condução do mapeamento, a string genérica de busca foi adaptada para cada base de dados e os resultados foram salvos em uma planilha no LibreOffice Calc. Ao todo 199 artigos foram encontrados. Todo o mapeamento aconteceu no período de 20 de Fevereiro a 22 de Maio de 2017.

Primeiramente foram removidos 35 artigos encontrados duplicados e, após leitura de título, resumo e palavras-chave na seleção inicial, foram aplicados os critérios de inclusão e exclusão que resultou em 65 artigos aceitos, sendo 99 recusados. Na segunda seleção foi feita uma leitura parcial (introdução, conclusão e os tópicos internos que responderiam as questões de pesquisa) dos artigos aceitos na primeira seleção. Nesta mesma etapa foram reaplicados os critérios de inclusão e exclusão obtendo 60 artigos aceitos no final (é possível ter acesso a estes trabalhos no link: "https://github.com/layonmartins/TrabalhosAceitosMapSistematico").

De todas as fontes de busca, a Scopus foi a que mais retornou trabalhos, com 143 (71,9\%), em segundo, o serviço de busca da ACM, retornou 25 (12,6\%). Uma observação foi que o serviço de busca da IEEE não retornou nenhum artigo com a string adaptada, porém percebeu-se que os artigos retornados pela Scopus incluíam artigos da IEEE, então optou-se por continuar a busca pela Scopus, sem utilizar o buscador da IEEE. A ScienceDirect retornou 17 (8,5\%), Springer Link 6 (3\%) (na Springer Link não foi possível aplicar filtros no título, resumo e palavras-chave diretamente no seu buscador, sendo feito posteriormente no Calc) e as demais fontes juntas (SBGames, SBIE, SVR), através de busca manual, retornaram $8(4 \%)$ trabalhos.

\section{Análise dos dados e resultados}

Nesta seção apresentamos algumas informações gerais, as respostas para as questões de pesquisa e algumas análises realizadas após a extração dos dados. $\mathrm{O}$ ano de publicação com mais trabalhos aceitos foi o de 2015 com 30 trabalhos (50\%), em segundo 2016 com 22 (36,7\%). O ano de 2017 até a data coletada (dia 08 de Março) foi de 3 artigos (5\%), a mesma quantidade publicada em 2014 e, por fim, o ano de 2013 com 2 (3,3\%). As Tabela 
VI Congresso Brasileiro de Informática na Educação (CBIE 2017)

Anais do XXVIII Simpósio Brasileiro de Informática na Educação (SBIE 2017)

5 e 6 apresentam uma relação de quantidades de trabalhos aceitos com os países e fontes de publicação em ordem decrescente.

Tabela 5. Relação de quantidade de trabalhos aceitos por países

\begin{tabular}{|l|l|}
\hline País & Quant. \\
\hline EUA & $9(13 \%)$ \\
\hline Brasil & $8(11,6 \%)$ \\
\hline Alemanha & $4(5,8 \%)$ \\
\hline Austrália, China, Índia, México, Polônia, Portugal e Reino Unido. & $3(4,3 \%)($ Cada um) \\
\hline Arábia Saudita, Espanha, Finlândia, Itália, Japão e Noruega. & $2(2,9 \%)($ Cada um) \\
\hline $\begin{array}{l}\text { Brunei Darussalam, Chipre, Coreia do Sul, Dinamarca, França, Grécia, Irlanda, Israel, Não identificado, } \\
\text { Nova Zelândia, Rússia, Suécia, Tailândia, Taiwan, Tunísia. }\end{array}$ & $1(1,4 \%)($ Cada um) \\
\hline
\end{tabular}

Tabela 6. Relação de quantidade de trabalhos aceitos por fontes de publicação

\begin{tabular}{|l|l|}
\hline Fonte & Quant. \\
\hline IEEE & $20(33,3 \%)$ \\
\hline ACM & $13(21,7 \%)$ \\
\hline Elsevier & $9(15 \%)$ \\
\hline Dechema e.V. & $3(5 \%)$ \\
\hline ASME e SBIE. & $2(3,3 \%)(\mathrm{Cada}$ um) \\
\hline $\begin{array}{l}\text { I.A.A.R.C., IAF, Academic Conferences Limited, American Chemical Society, American Society for En- } \\
\text { gineering Education, ASCE, Asian Association on Remote Sensing, IADIS, Maruzen Co., Ltd., SME e } \\
\text { Taylor and Francis Ltd. }\end{array}$ & $1(1,7 \%)(\mathrm{Cada}$ um) \\
\hline
\end{tabular}

\section{QP1. Qual óculos de RV foi utilizado?}

Os óculos de RV que mais se destacaram foram o "Oculus Rift", adotado em $43(65,2 \%)$ dos trabalhos analisados, o "Google Cardboard", com 8 (12,1\%), e o "Samsung Gear VR", com $4(6,1 \%)$. Conforme mostra a Figura 1, os outros modelos tiveram um índice bem inferior. Outra informação importante a se relatar sobre os óculos de RV são seus preços, até a data deste trabalho nos sites oficiais o preço em dolar se encontrava: Google Cardboard - U\$ 15,00; Samsung Gear - U\$ 93,99 e Oculus Rift - U\$ 399,00.

Figura 1. Óculos de RV utilizados

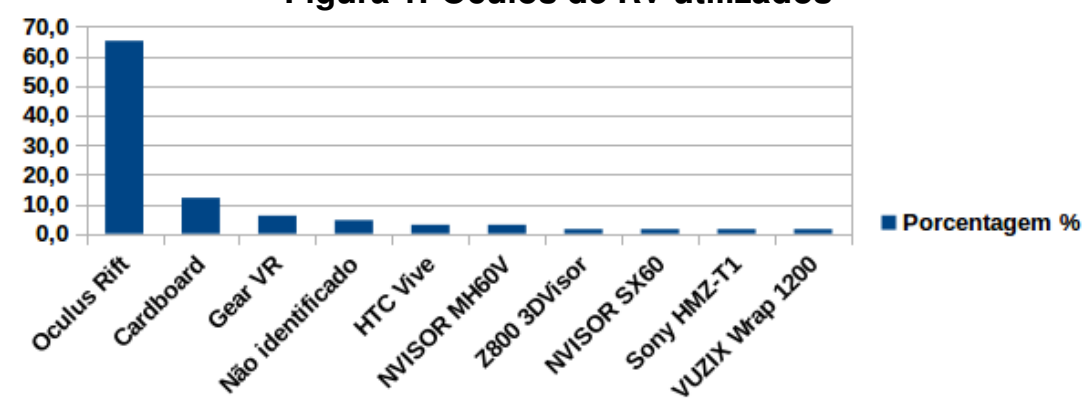

Fonte: Elaborado pelo autor.

\section{QP2. Qual software foi utilizado para desenvolver a aplicação de RV?}

O Software mais utilizado para desenvolver as aplicações foi a "Unity - Game Engine", utilizada em 28 (35,9\%) dos trabalhos analisados. Em segundo, o "SketchUP", foi utilizado em 6 trabalhos $(7,7 \%)$, e em terceiro, empatados, "Blender" e "Unreal Engine", cada um apareceu em $5(6,4 \%)$. Vale ressaltar que em 21 artigos (26,9\%), não foi possível identificar algum software ou ferramenta utilizada e o restante dos trabalhos $(16,7 \%)$ utilizaram outros software em menores índices (AutoCad, Photoshop, Maya, SketchFab etc). 
VI Congresso Brasileiro de Informática na Educação (CBIE 2017)

Anais do XXVIII Simpósio Brasileiro de Informática na Educação (SBIE 2017)

\section{QP3. Foram utilizados dispositivos além dos óculos de RV para melhorar a interação?}

Para interação foram encontrados vários dispositivos utilizados em conjunto com óculos e $\mathrm{RV}$, os que mais se destacaram foram o "joystick", utilizado em $12(16,2 \%)$ trabalhos analisados; o "Kinect" em 10 (13,5\%) e "Teclado e mouse" em 7 (9,5\%). Outros dispositivos encontrados também são mostrados na Figura 2.

Figura 2. Dispositivos de Interação utilizados

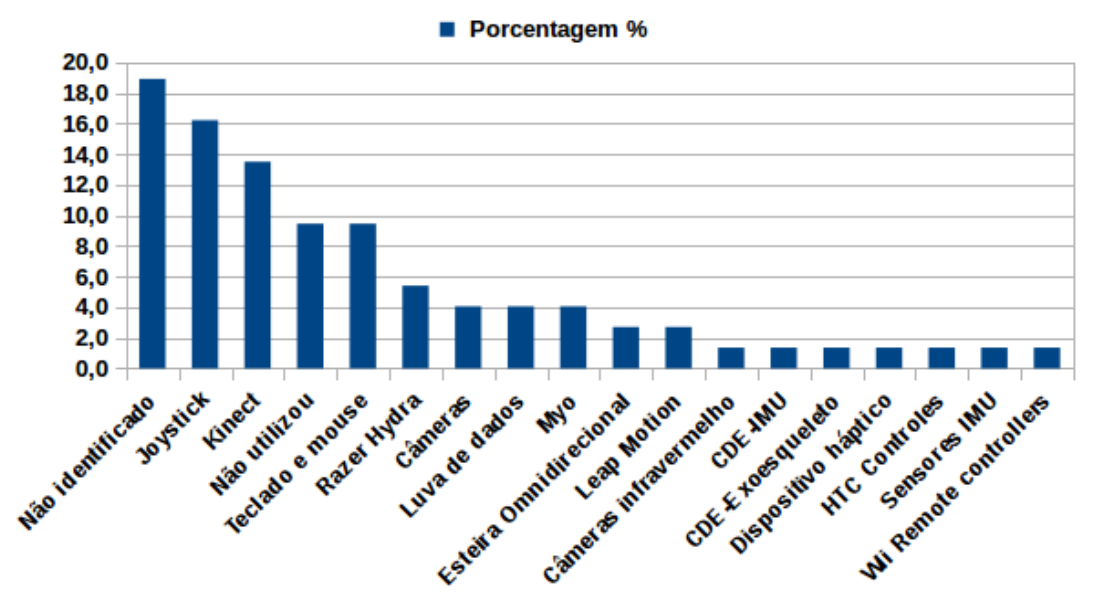

Fonte: Elaborado pelo autor.

\section{QP4. Quais técnicas de interação foram utilizadas?}

Várias formas de interação foram identificadas. Para simplificar a apresentação dos resultados desta questão de pesquisa, foi feita uma classificação das técnicas que são detalhadas na Tabela 7, contendo o nome da técnica, uma explicação geral do conceito proposto, quantidade de artigos e porcentagem. Alguns trabalhos utilizam mais de uma técnica de interação. Em 18 artigos $(22,2 \%)$ não foram possíveis identificar a técnica de interação utilizada.

\section{QP5. Qual área de aplicação?}

Encontrou-se uma variedade de aplicações RV em diversas áreas da educação e treinamento conforme pode ser visualizado na Figura 3.

As áreas que mais se destacaram foram Engenharias (Mecânica, Civil, Arquitetura etc.) com 12 (20\%) trabalhos e também a área de Saúde (Anatomia, Medicina, Enfermagem etc.) com $11(18,3 \%)$ trabalhos.

\section{QP6. Foram utilizados métodos de avaliação?}

A maioria dos trabalhos, 37 (61,7\%), apresentaram uma avaliação do sistema de RV proposto e $23(38,3 \%)$ não apresentaram. Todos que apresentaram uma avaliação descreveram uma avaliação empírica com usuários (crianças, estudantes e profissionais). Destes 37 trabalhos, em 19 foram identificados recursos (questionários, escala likert e quiz) para conduzir a avaliação. Percebeu-se também que 13 trabalhos realizaram comparações entre a aplicação desenvolvida com óculos de RV com outros sistemas não RV de ensino, 
VI Congresso Brasileiro de Informática na Educação (CBIE 2017)

Anais do XXVIII Simpósio Brasileiro de Informática na Educação (SBIE 2017)

Tabela 7. Técnicas de interação

\begin{tabular}{|c|c|c|}
\hline Técnica & Explicação & Quant. \\
\hline Botão físico & $\begin{array}{l}\text { Todo tipo de interação que usa algum tipo de botão físico ou controles, como botão } \\
\text { capacitivo do "Cardboard", Touch do "Gear VR", joystick etc. }\end{array}$ & $\begin{array}{l}21 \text { artigos } \\
(25,9 \%)\end{array}$ \\
\hline Manipulação natural & Utiliza movimentos naturais da mão para movimentar algum objeto dentro do MV. & $\begin{array}{l}11 \text { artigos } \\
(13,6 \%)\end{array}$ \\
\hline Gesto & $\begin{array}{l}\text { De forma geral são técnicas que utilizam algum tipo de movimentação natural do corpo } \\
\text { humano (um aceno) para interagir com o sistema mas que não são de manipulação ou } \\
\text { locomoção natural. }\end{array}$ & $\begin{array}{l}10 \text { artigos } \\
(12,3 \%)\end{array}$ \\
\hline Convencional & $\begin{array}{l}\text { Utilizam simplesmente o teclado e mouse convencionais, para navegar, selecionar e ma- } \\
\text { nipular o MV. }\end{array}$ & $\begin{array}{l}10 \text { artigos } \\
(12,3 \%)\end{array}$ \\
\hline Locomoção natural & $\begin{array}{l}\text { Técnica de navegação ou locomoção que tem a finalidade de fazer o usuário "andar" } \\
\text { dentro do MV semelhante ao caminhar natural (movimentos das pernas). }\end{array}$ & $\begin{array}{l}7 \text { artigos } \\
(8,6 \%)\end{array}$ \\
\hline Manipulação Háptica & $\begin{array}{l}\text { Técnica de manipulação de objetos virtuais que utiliza algum dispositivo háptico capaz } \\
\text { exercer força como resposta ao usuário. }\end{array}$ & $\begin{array}{l}1 \text { artigo } \\
(1,2 \%)\end{array}$ \\
\hline Raio de apontamento & $\begin{array}{l}\text { Técnica mais específica de seleção, que utiliza uma metáfora de raio para alcançar o } \\
\text { objeto a ser manipulado. }\end{array}$ & $\begin{array}{l}1 \text { artigo } \\
(1,2 \%)\end{array}$ \\
\hline Teletransporte & $\begin{array}{l}\text { Técnica mais específica de navegação, onde o usuário é capaz de se locomover no MV } \\
\text { de forma mais rápida, sem precisar "andar", ou seja, se teletransportando. }\end{array}$ & $\begin{array}{l}1 \text { artigo } \\
(1,2 \%)\end{array}$ \\
\hline Botão virtual & $\begin{array}{l}\text { Utiliza um tipo de botão que está presente apenas dentro do MV sendo capaz de realizar } \\
\text { alguma interação quando acionado pelo o usuário. }\end{array}$ & $\begin{array}{l}1 \text { artigo } \\
(1,2 \%)\end{array}$ \\
\hline
\end{tabular}

Figura 3. Áreas de educação e treinamento

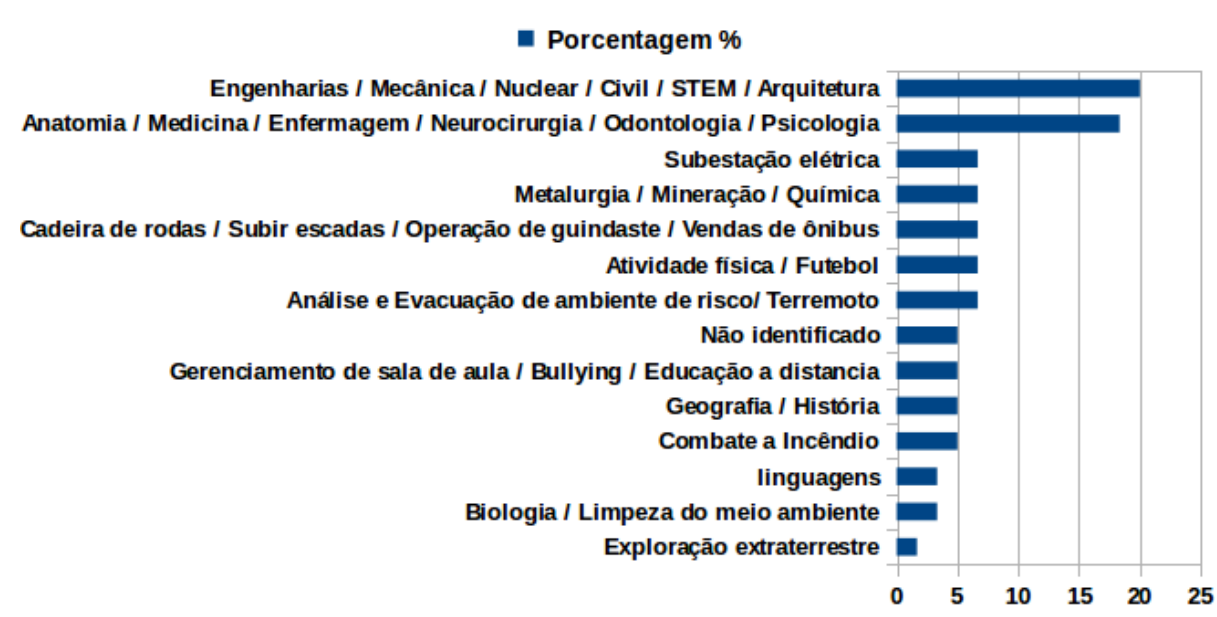

Fonte: Elaborado pelo autor.

destes 8 compararam uma aplicação RV, aplicada a um grupo de usuários, e uma segunda versão da aplicação sem RV, com monitor convencional, aplicada a outro grupo, ou ao mesmo grupo, respondendo os mesmos questionários e comparando os resultados. Dos 37 trabalhos que apresentaram uma avaliação, em 9 não foi possível identificar recursos ou técnicas utilizadas na avaliação.

Dos 13 trabalhos que apresentaram uma avaliação comparando os sistemas RV com não-RV, todos obtiveram resultados satisfatórios informando que a RV contribui para o aprendizado do aluno. [Ray and Deb 2016] e [Rasheed et al. 2015] comentam que a RV pode melhorar significativamente o interesse do aluno pelo conteúdo ensinado. [Alhalabi 2016] comenta que melhora o desempenho. [Tsaramirsis et al. 2016] comentam que os alunos preferem aprender o conteúdo das matérias com auxílio da RV. [Ebert et al. 2016] comentam que utilizando a RV os alunos "gravam" o conteúdo ensinado por mais tempo. 
VI Congresso Brasileiro de Informática na Educação (CBIE 2017)

Anais do XXVIII Simpósio Brasileiro de Informática na Educação (SBIE 2017)

\section{Discussão e Conclusão}

Com o mapeamento sistemático, percebeu-se que com o advento dos óculos de RV de baixo custo a partir de 2014, houve um grande aumento no número de pesquisas envolvendo o uso dessas tecnologias na educação. Isso mostra um bom interesse de pesquisadores na área, fator muito positivo para o aperfeiçoamento da tecnologia. Todos os trabalhos que apresentaram uma avaliação do sistema proposto concluíram que a RV contribui para uma melhor aprendizagem. Alguns trabalhos avaliaram uma aplicação com óculos de RV comparada a outra sem RV e depois, analisando os questionários respondidos com os mesmos conteúdos pelos diferentes grupos, percebeu-se que os grupos que utilizaram RV obtiveram melhores desempenhos.

Percebeu-se também uma grande variedade de aplicações educacionais e de treinamento. Muitos autores relataram a característica de segurança, sendo que principalmente nas aplicações de treinamento em ambientes de risco, o uso de RV contribui para a segurança dos usuários (por exemplo, aplicações de treinamento de combate a incêndio, subestação elétrica, mineração etc).

Fazendo uma classificação mais geral sobre as formas de interação, alguns trabalhos apresentam uma interação "natural" (baseada diretamente nos movimentos naturais do corpo humano, como gestos, movimentar braços e pernas para interagir dentro do AV 3D) [Tori et al. 2016], [Ha et al. 2016], [Hoang et al. 2016] etc. E outros uma interação "não-natural" (onde o usuário consegue interagir sem necessariamente movimentar seu corpo de forma natural, por exemplo, sentado em uma cadeira o usuário consegue locomover um avatar dentro do AV 3D utilizando botões, joysticks, teclado ou mouse, sem estar realmente movimentando suas pernas) [Ray and Deb 2016], [Cordeiro et al. 2015], [Moreira et al. 2016] etc.

Interações naturais forneceram uma maior sensação de imersão prendendo mais a atenção do usuário. Alguns trabalhos que testaram uma aplicação RV apenas com interação não-natural, não obtiveram resultados satisfatórios. Por parte dos usuários, uma interação natural pode fornecer melhor imersão, como apresentado em [Bharathi and Tucker 2015], porém segundo [Gong et al. 2015], quando é necessário precisão, as interações não-naturais são melhores. Por exemplo, ao se locomover usando uma interação natural o usuário teria que movimentar as pernas com precisão, caso contrário, com movimento errado não seria possível realizar a ação. Já com uma interação nãonatural bastaria pressionar um botão de um joystick.

Cordeiro et al. (2015) destacam que usuários que não têm familiaridade com um joystick ou teclado e mouse têm dificuldade em utilizar uma aplicação RV com interação não-natural, pois com o uso do óculos de RV perde-se o retorno visual das mãos e isso impede que os usuários vejam quais botões estão pressionando. Por outro lado, [Gorski et al. 2015] questiona que a utilização da interação natural é mais difícil de se aprender e que a interação não-natural é mais fácil e eficaz com menos tempo de aprendizagem. Isso pode ser levado em consideração para usuários que já estão acostumados com os controles convencionais da interação não-natural, sendo assim mais difícil de aprender a interagir com gestos e movimentos naturais do corpo humano.

Em resumo, os dados extraídos do Mapeamento Sistemático forneceram uma visão geral sobre o assunto, o quanto a RV (especialmente óculos de RV de baixo custo) 
VI Congresso Brasileiro de Informática na Educação (CBIE 2017)

Anais do XXVIII Simpósio Brasileiro de Informática na Educação (SBIE 2017)

vem sendo aplicada à educação, suas formas de interação, áreas de aplicação e formas de avaliação destes trabalhos. Poucos destes trabalhos analisaram as técnicas de interação dos óculos de RV de baixo custo, que têm potencial de maior adesão em contextos educacionais, apesar de terem opções de interação limitadas. Por exemplo, o Google Cardboard por se tratar do modelo de óculos de mais baixo custo que os demais, é mais limitado quanto a interação, possuindo apenas um botão (existem modelos que não têm este botão) para interagir com todo o sistema de RV (locomoção, seleção e manipulação), desta forma sendo necessário outras técnicas de interação.

Conclui-se que a RV contribui positivamente para o desenvolvimento de novas aplicações educacionais, melhorando o aprendizado dos usuários. Também conforme relata [Kleven et al. 2014] a RV não vem para substituir a forma tradicional de aula ou aplicações, mas sim como um complemento, pois ainda há usuários que preferem aprender com aplicações sem RV, por sentirem algum mal-estar ao usar os óculos de RV ou apenas por motivo pessoal. Enfim, espera-se que este mapeamento sistemático possa contribuir com uma análise sobre o quanto e como a RV, especificamente as aplicações baseadas em óculos RV, tem sido aplicada à educação.

\section{Referências}

Alhalabi, W. (2016). Virtual reality systems enhance students achievements in engineering education. Behaviour and Information Technology, 35(11):919-925.

Bharathi, A. K. B. G. and Tucker, C. S. (2015). Investigating the impact of interactive immersive virtual reality environments in enhancing task performance in online engineering design activities. In ASME 2015 International Design Engineering Technical Conferences and Computers and Information in Engineering Conference, pages 1-11. American Society of Mechanical Engineers.

Brereton, P., Kitchenham, B. A., Budgen, D., Turner, M., and Khalil, M. (2007). Lessons from applying the systematic literature review process within the software engineering domain. Journal of Systems and Software, 80(4):571 - 583. Software Performance.

Cordeiro, C., Paludo, J., Tanaka, E., Domingues, L., Gadbem, E., and Euflausino, A. (2015). Development of immersive virtual reality environment to train electricians to work on substations. In 2015 XVII Symposium on Virtual and Augmented Reality, pages $142-146$.

Davis, S., Nesbitt, K., and Nalivaiko, E. (2014). A systematic review of cybersickness. In Proceedings of the 2014 Conference on Interactive Entertainment, IE2014, pages 8:1-8:9, New York, NY, USA. ACM.

Ebert, D., Gupta, S., and Makedon, F. (2016). Ogma - a virtual reality language acquisition system. volume 29-June-2016.

Gong, X., LIU, Y., JIAO, Y., WANG, B., ZHOU, J., and YU, H. (2015). A novel earthquake education system based on virtual reality. IEICE Transactions on Information and Systems, E98.D(12):2242-2249.

Gorski, F., Bun, P., Wichniarek, R., Zawadzki, P., and Hamrol, A. (2015). Immersive city bus configuration system for marketing and sales education. Procedia Computer Science, 75:137 - 146. 2015 International Conference Virtual and Augmented Reality in Education. 
VI Congresso Brasileiro de Informática na Educação (CBIE 2017)

Anais do XXVIII Simpósio Brasileiro de Informática na Educação (SBIE 2017)

Ha, G., Lee, H., Lee, S., Cha, J., and Kim, S. (2016). A vr serious game for fire evacuation drill with synchronized tele-collaboration among users. volume 02-04-November2016, pages 301-302.

Hoang, T., Reinoso, M., Vetere, F., and Tanin, E. (2016). Onebody: Remote posture guidance system using first person view in virtual environment. volume 23-27-October2016.

Kawakami, R., d. S. Nunes, E. P., and Maciel, C. (2015). Three-dimensional virtual environments that support hospitalized children's learning: A systematic review. In 2015 XVII Symposium on Virtual and Augmented Reality, pages 190-199.

Kitchenham, B. and Charters, S. (2007). Guidelines for performing systematic literature reviews in software engineering. In Technical report, Ver. 2.3 EBSE Technical Report. EBSE. sn.

Kleven, N. F., Prasolova-FÃşrland, E., Fominykh, M., Hansen, A., Rasmussen, G., Sagberg, L. M., and Lindseth, F. (2014). Training nurses and educating the public using a virtual operating room with oculus rift. In 2014 International Conference on Virtual Systems Multimedia (VSMM), pages 206-213.

Moreira, P., de Oliveira, E. C., and Tori, R. (2016). Impact of immersive technology applied in computer graphics learning. In Brazilian Symposium on Computers in Education (Simpósio Brasileiro de Informática na Educação-SBIE), volume 27, page 410.

Pantelidis, V. S. (2009). Reasons to use virtual reality in education and training courses and a model to determine when to use virtual reality. Themes in Science and Technology Education, 2:59-70.

Proenca, A. P., Neto, M. M., Domingues, R. G., Borges, L. R., Cardoso, A., and Notargiacomo, P. (2017). Influence degree analysis of the emphases of education and training in virtual environments dimensional learning. IEEE Latin America Transactions, 15(5):974-980.

Rasheed, F., Onkar, P., and Narula, M. (2015). Immersive virtual reality to enhance the spatial awareness of students. volume 17-19-December-2015, pages 154-160.

Ray, A. and Deb, S. (2016). Smartphone based virtual reality systems in classroom teaching - a study on the effects of learning outcome. pages 68-71.

Tori, R., Wang, G., Sallaberry, L., de Oliveira, E. C., Machado, M. A. d. A. M., and Tori, A. A. (2016). Treinamento odontológico imersivo por meio de realidade virtual. In Brazilian Symposium on Computers in Education (Simpósio Brasileiro de Informática na Educação-SBIE), volume 27, page 400.

Tsaramirsis, G., Buhari, S., Al-Shammari, K., Ghazi, S., Nazmudeen, M., and Tsaramirsis, K. (2016). Towards simulation of the classroom learning experience: Virtual reality approach. pages 1343-1346. 